\title{
Application values of prenatal screening and non-invasive gene sequencing in fetal birth defects
}

\author{
Jingjing Bu ${ }^{1}$, Pan Jiang ${ }^{2}$, Xiaoli Cui ${ }^{3}$, \\ Hongyan $\mathrm{Zhou}^{4}$, Fengxia Han ${ }^{5}$
}

\begin{abstract}
Objective: To investigate effects of prenatal screening and non-invasive gene sequencing on the clinical diagnosis of fetal birth defects and the outcome of pregnancy.

Methods: Totally 2520 pregnant women who received prenatal screening in our hospital were selected as the research subjects. The high-risk pregnant women were further tested by the non-invasive gene sequencing technology. Pregnant women with positive results were diagnosed by amniocentesis and fetal chromosome karyotype analysis, and the pregnancy outcome was followed up for one year.

Results: 870 out of the 2520 pregnant women was tested by non-invasive gene sequencing technology; 26 of the 870 women was 13 -trisomy-positive and was diagnosed by amniocentesis and fetal chromosome karyotype analysis, 22 of which was diagnosed as $47, \mathrm{XN},+13$ and four of which was normal; the diagnosis accuracy of non-invasive prenatal testing (NIPT) was $84.6 \% .18$ out of the 22 confirmed cases underwent abortion, three cases had termination of embryonic development, and one case had postnatal anomaly. Thirty four out of the 2520 pregnant women was 18-trisomy-positive and was diagnosed by amniocentesis and fetal chromosome karyotype analysis, 31 of which was diagnosed as $47, \mathrm{XN},+18$ and three cases were normal; the diagnosis accuracy of NIPT was $91.2 \%$. 29 out of the 31 confirmed cases underwent abortion and two cases had termination of embryonic development. Forty out of the 2520 pregnant women was 21-trisomy-positive and was diagnosed by amniocentesis and fetal chromosome karyotype analysis, 39 of which was diagnosed as $47, \mathrm{XN},+21$ and one case was normal; the diagnosis accuracy of NIPT was $97.5 \%$. Thirty four out of the 39 confirmed cases underwent abortion, three cases had termination of embryonic development, and two cases had postnatal anomaly. Twenty eight cases were tested as sex chromosomepositive and were diagnosed by amniocentesis and fetal chromosome karyotype analysis, 25 out of which was diagnosed as abnormal and three cases were normal; the diagnosis accuracy of NIPT was $89.3 \%$. 24 out of the 25 confirmed cases underwent abortion, and one case had termination of embryonic development.

Conclusion: Prenatal screening and non-invasive gene sequencing technology have a high accuracy in the diagnosis of fetal birth defects, which can reduce the maternal abortion injury as much as possible and relieve the psychological pressure. The promotion of the mode can be strengthened in clinics.
\end{abstract}

KEYWORDS: Prenatal screening; Non-invasive gene sequencing; Fetal birth defects; Pregnancy outcome.

How to cite this:

doi: https://doi.org/10.12669/pjms.36.7.2290

$B u J$, Jiang $P$, Cui $X$, Zhou $H$, Han $F$. Application values of prenatal screening and non-invasive gene sequencing in fetal birth defects. Pak J Med Sci. 2020;36(7):1545-1549. doi: https://doi.org/10.12669/pjms.36.7.2290

This is an Open Access article distributed under the terms of the Creative Commons Attribution License (http://creativecommons.org/licenses/by/3.0), which permits unrestricted use, distribution, and reproduction in any medium, provided the original work is properly cited.

Correspondence:

Jingjing $\mathrm{Bu}$,

No.515 Huanghe $7^{\text {th }}$ Road,

Binzhou People's Hospital, Shandong,

256600, China.

E-mail: bujingjingo@163.com

* Received for Publication:

* Revision Received:

* Revision Accepted:
January 28, 2020

June 16, 2020

June 20, 2020

\section{INTRODUCTION}

Birth defects mainly refer to congenital diseases such as body structure, function or metabolism abnormality before birth, which is the main cause of infant death and congenital disability in clinic. ${ }^{1,2}$ According to statistics, the current incidence of birth defects in China is about $4 \% \sim 6 \%$. In recent 
years, due to the increase of the birth rate of the second child, the incidence of birth defects has a significant increase trend. ${ }^{3}$ Chromosomal disease is the main disease of fetal birth defects, including 13-trisomy syndrome, 18-trisomy syndrome and 21-trisomy syndrome. ${ }^{4,5}$

At present, there is no effective treatment for this kind of disease, which can only be diagnosed and prevented in time by prenatal screening and non-invasive gene sequencing technology. ${ }^{6}$

Noninvasive DNA detection is to sequence the free DNA fragments in maternal peripheral plasma by using a new generation of DNA sequencing technology by extracting the venous blood of pregnant women and analyze the biological information of the sequencing results, from which fetal genetic information can be obtained; the genome coverage rate is as high as $93 \%$.

Ji pointed out that non-invasive gene sequencing technology as the main detection technology could improve the efficiency of prenatal examination in the development of prenatal examination of pregnant women. ${ }^{7} \mathrm{He}$ found that the noninvasive gene sequencing technology could analyze the related diseases according to the DNA gene sequence fragment provided by pregnant women, scientifically identify the growth status of the fetus in the maternity examination, and analyze the fetal trisomy 21 syndrome, trisomy 18 syndrome, monosomy 18 syndrome, trisomy 13 syndrome, and $\mathrm{X}$ chromosome and $\mathrm{Y}$ chromosome abnormalities, i.e., the technology could meet the practical needs of fetal birth defects detection. Feng et al. found that the most significant feature of non-invasive gene sequencing technology was that the harm to pregnant women was reduced to the minimum or even zero and pregnant women had no pain in the detection process. ${ }^{8}$ Based on this, the authors selected 2520 pregnant women in the second trimester who underwent prenatal screening in our hospital in the past one year as the research subjects, carried out non-invasive gene sequencing technology and amniocentesis chromosome karyotype diagnosis for those with abnormal results, and analyzed the clinical effect of the diagnosis results on the prevention of fetal birth defects, as well as the impact on pregnancy outcome.

\section{METHODS}

Two thousand two hundred fifty pregnant women in the second trimester who underwent prenatal screening from April 2018 to May 2019 were selected as the main subjects of study. All pregnant women were singleton, have been pregnant for 17-20 weeks, have gave birth for one to three times (average $(2.3 \pm 0.9)$ times), and aged $18-47$ years old (average $(28.17 \pm 2.35)$ years old). The detection subjects included pregnant women who showed with high risk, critical risk and single value abnormality in Down's syndrome screening, showed nuchal translucency (NT) thickening in ultrasonic examination, were elderly, have exceeded the gestational age, or were required to receive examination. Family members of the fetus were informed with the study protocol and were willing to take relevant risks. They have signed the informed consent for the study, and the study was approved by the medical ethics committee (Ref.No. \# 161, Dated January 14, 2020).

Methods: Prenatal screening: 2-3 mL of fasting venous blood was extracted from each pregnant woman. The alpha fetoprotein (AFP), free estriol $(\mathrm{uE} 3)$ and free- $\beta$ human chorionic gonadotrophin (free- $\beta \mathrm{HCG}$ ) in the serum was screened using time-resolution fluorescence immunoassay analyzer (Nanjing Lansion Biotechnology Co., Ltd.; model type: LS-2150). The risk values of fetal Patau syndrome (T13), Edwards's syndrome (T18) and Down's syndrome (T21) were evaluated by Multiaale software combined with the basic information of pregnant women, such as weight, gestational age and age.

Noninvasive gene sequencing technology (NIPT): $15 \mathrm{~mL}$ of fasting venous blood was taken from each woman and tested by a third-party sequencing company cooperating with our hospital. After separating the plasma, the free DNA fragments (including fetal free DNA) in the maternal peripheral plasma were sequenced by a new generation of DNA sequencing technology, and the sequencing results were analyzed for biological information, from which the genetic information of the fetus could be obtained, so as to judge whether the fetus was suffering from chromosomal diseases. Amniocentesis and karyotype analysis: before amniotic fluid extraction, the pregnant women emptied their bladder and lay on their back at a designated position. The deepest part of amniotic fluid was identified through B-ultrasound and marked. After strict disinfection, a lumbar puncture needle was slowly punctured into the marked part and slowly withdrawn after reaching the amniotic cavity of the uterus. Then two $\mathrm{mL}$ of amniotic fluid which was extracted 
Prenatal screening \& non-invasive gene sequencing in fetal birth defects

Table-I: Detection results of non-invasive gene sequencing technology [n (\%)].

\begin{tabular}{lcccc}
\hline Types of risks for pregnant women & Critical risk & High risk & Elder age & Total \\
\hline Number of cases & 379 & 152 & 339 & 870 \\
13-trisomy-positive & $1(0.26)$ & $24(15.79)$ & $1(0.29)$ & $26(2.99)$ \\
18-trisomy-positive & $1(0.26)$ & $28(18.42)$ & $5(1.47)$ & $34(3.91)$ \\
21-trisomy-positive & $3(0.79)$ & $26(17.11)$ & $11(3.24)$ & $40(4.60)$ \\
Sex chromosome-positive & $2(0.53)$ & $22(14.47)$ & $4(1.18)$ & $28(3.22)$ \\
\hline
\end{tabular}

for the first time was discarded, and then $20 \mathrm{~mL}$ of amniotic fluid was collected by two tubes, 20 $\mathrm{mL}$ each tube. The amniotic fluid was centrifuged, the supernatant was discarded, the remaining amniotic fluid cells were cultured in vitro for preparation of chromosomes, and then the fullautomatic intelligent chromosome karyotype analysis system (IMSTAR company, France, model type, Meta Scan Karyotyping System) was used for karyotype analysis.

Determination criteria: According to the medical authentication, primiparas over 35 years old were pregnant woman at an elder age, and old age is one of the high-risk factors. The risk values of chromosomal diseases are divided into low risk value, critical risk value and high risk value. 13-trisomy below $1 / 150$ is the low-risk value, $1 / 150 \sim 1 / 1000$ is the critical risk value, $1 / 150$ or higher is the high-risk value, i.e., positive. 18 -trisomy below $1 / 350$ is the low-risk value, $1 / 350 \sim 1 / 1000$ is the critical risk value, $1 / 350$ or higher is the high-risk value, i.e., positive. 21 -trisomy below $1 / 270$ is the low-risk value, $1 / 270 \sim 1 / 500$ is the critical risk value, and 1/270 or higher is the high risk value, i.e., positive. Critical and high-risk pregnant women and pregnant women at an elder age were tested by non-invasive gene sequencing technology.

\section{RESULTS}

Two thousand two hundred fifty pregnant women underwent prenatal screening, and pregnant women at an elder age accounted for $13.45 \%(339 / 2520)$. The prenatal screening found that pregnant women with low risk value accounted for $65.48 \%(1650 / 2520)$, pregnant women with critical risk value accounted for $15.04 \%(379 / 2520)$, and pregnant women with high risk value accounted for $6.03 \%(152 / 2520)$.

The detection results of the non-invasive gene sequencing technology showed that the prevalence rate was $14.7 \%(128 / 870)$. Among the 379 pregnant women with critical-risk value, one case was 13-trisomy syndrome-positive, one case was 18-trisomy syndrome-positive, four cases were 21-trisomy syndrome-positive, and two cases were sex chromosome syndrome-positive. Among 152 pregnant women with high risk value, 24 cases were positive for 13-trisomy syndrome, 28 cases were 18-trisomy syndrome-positive, 26 cases were 21-trisomy syndrome-positive, and 22 cases were sex chromosome syndromepositive. Among the pregnant women at an elder age, one case had 13-trisomy syndrome-positive, five cases were 18-trisomy syndrome-positive, ten cases were positive 21-trisomy syndromepositive, and three cases were sex chromosome syndrome-positive (Table-I).

The results of amniocentesis and karyotype analysis showed that the prevalence rate was 13.4\% $(117 / 870)$. Among twenty-six pregnant women who showed a positive NIPT result for 13-trisomy, 22 of them were confirmed as $47, \mathrm{XN},+13$ by amniocentesis and karyotype analysis, and four of them were normal. Among thirty-four pregnant women who showed a positive NIPT result for 18 -trisomy, 31 of them were confirmed as $47, \mathrm{XN}$, +18 by amniocentesis and karyotype analysis, and three of them were normal. Among forty pregnant women who showed a positive NIPT

Table-II: Comparison between NIPT results and results of amniocentesis and karyotype analysis [n (\%)].

\begin{tabular}{lccc}
\hline NIPT results & No. of cases & No. of confirmed cases & Diagnosis accuracy of NIPT \\
\hline 13-trisomy-positive & 26 & 22 & 84.62 \\
18-trisomy-positive & 34 & 31 & 91.18 \\
21-trisomy-positive & 40 & 39 & 97.50 \\
Sex chromosome-positive & 28 & 25 & 89.29 \\
\hline Total & 128 & 117 & $/$ \\
\hline
\end{tabular}


result for 21-trisomy, nine cases were confirmed as $47, \mathrm{XN},+21$ by amniocentesis and karyotype analysis, and one case was normal. Among twenty-eight pregnant women who showed a positive NIPT result for sex chromosome, 25 cases were confirmed as abnormal by amniocentesis and karyotype analysis, and three cases were normal (Table-II).

Pregnancy Outcome: After the diagnosis by amniocentesis and karyotype analysis, 18 out of 22 pregnant women with 13-trisomy syndrome underwent abortion, three women selected termination of fetal development, and one case had abnormal fetus after birth; 29 out of 31 pregnant women with 18-trisomy syndrome underwent abortion, and two women selected termination of fetal development; 34 out of 39 pregnant women with 21-trisomy syndrome underwent abortion, three women selected termination of fetal development; 24 out of the 25 pregnant women with sex chromosome syndrome underwent abortion, and one women selected termination of fetal development (Table-III).

\section{DISCUSSION}

In China, birth defects caused by chromosomal diseases occur every year, and the incidence is increasing year by year. ${ }^{9}$ The most common prenatal screening in clinic is to extract AFP, $\mathrm{uE3}$ and free- $\beta$ HCG from the serum of pregnant women, calculate the risk value of chromosomal abnormality with Multiaale software, and screen out the critical high-risk group or high-risk group that may have some kind of chromosomal disease, so as to improve the accuracy of detection. ${ }^{10,11}$

Traditional prenatal diagnosis techniques include amniocentesis, chorionic villus biopsy, fetal umbilical vein puncture and fetal tissue biopsy, but it can cause bleeding, with $0.5 \%-1 \%$ risk of complications such as infection and abortion. ${ }^{12}$ It brings great psychological pressure to pregnant women and their families and greatly reduces the compliance of prenatal diagnosis.
In order to plan the work of maternity examination for pregnant women better, people are constantly changing the technology of maternity examination in the development of clinical medicine. The non-invasive gene sequencing technology, as the main technology in the prenatal examination of pregnant women, is a new form of technology practice formed in the development of existing medicine. Non-invasive gene sequencing technology has strong practicality in prenatal examination of pregnant women as it can detect various diseases and make scientific judgment on fetal development.

The results showed that there were 1650 lowrisk pregnant women, 379 critical-risk pregnant women, 152 high-risk pregnant women and 339 pregnant women at an elderly age, and 870 pregnant women might have abnormal factors and underwent non-invasive gene sequencing. Among the 870 pregnant women tested by NIPT, 26 cases were 13-trisomy-positive, 34 cases were 18-trisomy-positive, 40 cases were 21-trisomypositive, and 28 were sex chromosome positive, which showed that NIPT had a good diagnostic performance for fetal chromosomal diseases and could effectively improve the diagnostic accuracy of prenatal screening, and the conclusion was in line with a previous research. ${ }^{13}$ After that, the above-mentioned patients were diagnosed by amniocentesis and karyotype analysis. The results showed that the diagnosis rate was kept above $80 \%$, indicating that the detection accuracy of the gene sequencing technology was not $100 \%$, there were false-positive and false-negative, and amniocentesis and karyotype analysis were needed for further accurate diagnosis.

Limitations and Shortcomings: This study is limited to the limitations of time, research level and experimental conditions, and there were many deficiencies to be improved, for example, the cost comparison of traditional methods and NIPT was not included in the research results and the

Table-III: Pregnancy outcome [n (\%)].

\begin{tabular}{lccccc}
\hline $\begin{array}{l}\text { Diagnosis type of amniocentesis } \\
\text { and karyotype analysis }\end{array}$ & $\begin{array}{c}\text { No. of } \\
\text { cases }\end{array}$ & Abortion & $\begin{array}{c}\text { Termination of } \\
\text { fetal development }\end{array}$ & $\begin{array}{c}\text { Fetal normal } \\
\text { after birth }\end{array}$ & $\begin{array}{c}\text { Fetal abnormal } \\
\text { after birth }\end{array}$ \\
\hline 13-trisomy-positive & 22 & $18(81.82)$ & $3(13.64)$ & $0(0)$ & $1(4.54)$ \\
18-trisomy-positive & 31 & $29(93.55)$ & $2(6.45)$ & $0(0)$ & $0(0)$ \\
21-trisomy-positive & 39 & $34(87.18)$ & $3(7.69)$ & $0(0)$ & $2(5.13)$ \\
Sex chromosome-positive & 25 & $24(96)$ & $1(4)$ & $0(0)$ & $0(0)$ \\
\hline Total & 117 & $105(89.74)$ & $9(7.69)$ & $0(0)$ & $3(2.57)$ \\
\hline
\end{tabular}


differences between different ethnic groups were not considered, all of which need to be further discussed in the follow-up study.

\section{CONCLUSION}

In conclusion, the average detection rate of non-invasive gene sequencing technology for chromosomal syndrome is above $90 \%$, which is worth promoting and applying in clinic. But it cannot completely replace prenatal screening and diagnosis. According to clinical observation, prenatal screening can screen out high-risk chromosomal diseases and other diseases of pregnant women. Therefore, in order to improve eugenics and eugenics inheritance, this study suggests that prenatal screening and non-invasive gene sequencing technology should be combined with prenatal chromosome karyotype analysis to make a correct diagnosis of fetal birth defects, and mutual cooperation can reduce the blindness of screening fetal birth defects.

Declaration of interest: All authors declared there was no conflict interests involved.

\section{Grant Support E Financial Disclosures: None.}

\section{REFERENCES}

1. Fitzgerald B, Boyle C, Honein MA. Birth defects potentially related to zika virus infection during pregnancy in the United States. JAMA. 2018;319(12):11951196. doi: 10.1001/jama.2018.0126

2. Delaney A, Mai C, Smoots A, Cragan J, Ellington $\mathrm{S}$, Langlois $\mathrm{P}$, et al. Population-based surveillance of birth defects potentially related to zika virus infection - 15 states and U.S. Territories, 2016. MMWR Morb Mortal Wkly Rep. 2018;67(3):91-96. doi: 10.15585/mmwr. mm6703a2

3. Wu QM, Zhou J. Advances in prenatal screening and prenatal diagnosis for birth defect. China J Birth Health Hered. 2015;10:43-45.

4. Chen EZ, Chiu RW, Sun H, Akolekar R, Chan KCA, Leung TK, et al. Noninvasive prenatal diagnosis of fetal trisomy 18 and trisomy 13 by maternal plasma DNA sequencing. PLos One. 2011;6(7):e21791. doi: 10.1371/ journal.pone.0021791

5. He F, Hu X, Xiong W, Li L, Lin L, Shen B, et al. Directed bmp4 expression in neural crest cells generates a genetic model for the rare human bony syngnathia birth defect. Dev Biol. 2014;391(2):170-181. doi: 10.1016/j. ydbio.2014.04.013
6. Zhang B, Pan L, Wang H, Liu J, Lu B, Chen Y, et al. Performance of prenatal screening by non-invasive cell-free fetal DNA testing for women with various indications. China J Med Genet. 2018;35(1):51-55. doi: 10.3760/cma.j.issn.1003-9406.2018.01.011

7. Ji LL. Clinical diagnosis of prenatal birth defects by plasma free DNA high-throughput sequencing. Women's Health Res. 2017;11(5):156-158.

8. Feng XL, Shen H, Liu D, Luo SX, Li B, Dong RL, et al. The clinical application research of prenatal screening for fetus birth defects and noninvasive gene sequencing technology. China J Birth Health Hered. 2017;25(04):12-14.

9. Jin YX, Miao ZY, Ge JM, Zhang WH, Li SP, Liu XD Prenatal diagnosis of fetal chromosome aneuploidy by massively parallel genomic sequencing. Natl Med J China. 2014;94(23):1785-1787. doi: 10.3760/cma.j.is sn.0376-2491.2014.23.008

10. Vogel I, Petersen OB. Prenatal screening for atypical chromosomal abnormalities: Past or future? Ultrasound Obstet Gynecol. 2018;51(4):434-435. doi: 10.1002/ uog. 19030

11. Hou YP, Yang JX, Guo FF, Qi YM, Peng HS, Wang DM, et al. Application of non-invasive DNA prenatal detection in the fetal chromosomal aneuploidy screening. Lab Med Clin. 2018;15(11):1542-1544,1548. doi: 10.3969/j.issn.16729455.2018.11.002

12. Langlois S, Brock JA. Genetics Committee. Current status in non-invasive prenatal detection of down syndrome, trisomy 18, and trisomy 13 using cell-free DNA in maternal plasma. J Obstet Gynaecol Can. 2013;35(2):177181. doi: 10.1016/S1701-2163(15)31025-2

13. Wen $P$, Xue $Y$, Zhang Q, Liang Q, Li Q, Li H, et al. Two false negative cases in noninvasive prenatal testing for fetal chromosomal aneuploidies. China J Med Genet. 2017;34(6):884-887. doi: 10.3760/cma.j.is sn.1003-9406.2017.06.022

\section{Authors' Contribution:}

JJB \& PJ: Study design, data collection and analysis. XLC \& HYZ: Manuscript preparation, drafting and revising.

JJB \& FXH: Review and final approval of manuscript.

JJB is responsible and accountable for the accuracy and integrity of the work.

Authors:

1. Jingjing Bu

Central laboratory,

2. Pan Jiang

Central laboratory,

3. Xiaoli Cui

Central laboratory,

4. Hongyan Zhou

Clinical Laboratory,

5. Fengxia Han

Clinical Laboratory,

1-5: Binzhou People's Hospital, Shandong, 256600, China. 